\title{
Probabilistic load flow methods to estimate impacts of distributed generators on a LV unbalanced distribution grid
}

\author{
Fallilou DIOP \\ ( IRT SystemX,CentralSupelec,GEEPS) \\ Paris, France \\ fallilou.diop@irt-systemx.fr
}

\author{
Martin Hennebel \\ ( CentraleSupelec, GEEPS) \\ Paris,France \\ martin.hennebel@centralesupelec.fr
}

\begin{abstract}
The aim of this paper is to apply probabilistic load flow methods on a three phases, unbalanced low voltage distribution network. We use a point estimate method and a Monte Carlo simulation based method to estimate the electrical characteristics (buses voltage, phases and neutral conductors currents) of a distribution grid in presence of a large number of small size photovoltaic generators. Probabilistic load flow allows us to take into account the uncertainty of photovoltaic production and load consumption in load flow computation. The literature shows that PEM method gives good accuracy results while requiring less time simulation than Monte Carlo simulation. In this paper, we aim to check if this assumption is still right with different kinds of probability density function and for a large size electrical network. Usually, random parameters are modeled as a normal distribution. In this work, a generalized extreme value is used to model load consumption behaviour instead of a normal one. The uncertainty of photovoltaic production is supposed to be directly linked to the sky clear index which is modeled as a beta distribution.
\end{abstract}

Index Terms-Low voltage network, Monte Carlo simulation, Point estimate method, Probabilistic load flow

\section{INTRODUCTION}

Nowadays, the climate changes combined with oil's prices fluctuation lead to look for alternatives to fossil primary sources of energy. In this context actions are taken to promote use of renewable energy sources (PV, wind power, biomass...) that implies rise of injection from generators connected to the power distribution grid.

Electrical power system scheme is composed by production system, transmission and distribution networks. The distribution network plays an important role in this energy transition context as it is the interface between small and medium size power distributed generator and the rest of electrical system. The problem is, historically, for technical reasons, this distribution network was not designed to receive production units. So, currently, one existing challenge is to ensure good operating conditions of the electrical network in this presence of DGs by respecting the technical constraints standards (voltage limits, current limits, unbalance...).

For that, DSO need a good estimation of impacts of DG on those technical constraints which are directly linked to the network reliability. For the sake of convenience with reality, the estimation tool should be able to take into account the uncertainty of production and consumption at a given time. Probabilistic load flow concept is suitable to do it.

Load flow is used to determine voltage magnitude and phase angle at each node of the network under a given operating condition (load and generation profile). Usually, deterministic load flow is used but in that case, load and generation profiles at each node of the network are supposed to be exactly known at each time step; what does not take into account the uncertainty on production and consumption . That is why, in this paper, we plan to achieve load flow calculation taking into account the random aspect of the demand and production.

In the literature three main methods of probabilistic load flow are noticed:

The simulation based method known as MonteCarlo [1], [2]. It consists of solving a deterministic non linear load flow problem for several scenarios of power production and consumption. Scenarios are generated randomly from a probability density function. Although the MCs method might give accurate results, it requires a large number of simulation to converge.

The analytical methods [3] are based on the linearization of the power flow equation. These methods don't need long time simulation but the use of approximate equations implies a lack of accuracy.

An alternative approximation method called Point Estimate Method has been developed and applied in case of probabilistic load flow in presence of wind turbines. It solves the deterministic load flow at several sample points and then weights them to estimate the output moments [4].

In this paper, we will apply a point estimate method and Monte Carlo simulation method to estimate PV impacts on voltage, current, power and voltage unbalance of low voltage electrical network.

In the following sections, we first discuss impacts of PV on chosen technical constraints which are voltage, current, power and voltage unbalance. After that we describe the applied PLF method. Then, we present the simulation parameters: the network test model, the probabilistic load and production 
model. To finish, we compare results from PEM method to those obtained from the Monte Carlo one.

\section{IMPACT OF PV INTEGRATION ON LV DISTRIBUTION NETWORK}

Injection from DGs such as PV implies many technical impacts on power distribution network [5]. In our case, we have focused on impacts of PV generation units on nodes voltage, current intensity in phase and neutral conductors and voltage unbalance.

\section{A. Voltage rise issue}

The power produced by $\mathrm{PV}$ varies the power flows in the network, which impacts the voltage profile. In the AC power system, the voltage difference between two points can be approximated by (1) where $P$ is the active power, $Q$ the reactive power, $R, X$ respectively the line resistance and reactance and $V_{n}$ the mean voltage [6].

$$
\Delta V \approx \frac{P \times R+Q \times X}{V_{n}}
$$

In transmission network, voltage variation due to injection of active power can be ignored due to the fact that $X / R$ is high because of the low resistance value. The problem is more crucial in distribution network with higher value of $R$, so PV power injection tends to increase local voltage value.

\section{B. Over current and overcharge issue}

Over current can cause thermal problems due to the Joule effect. In case of low PV penetration, i.e when installed PV capacity is relatively small compared to consumption, the produced electricity is consumed locally and it reduces the consumption of the customer from the source and therefore the current amplitude is also reduced. However, with high penetration of PV, it is possible to have a backward power flow. The worst case is when the difference between PV production and consumption, exceeds the line or local transformer capacity. Over current increases the lines and transformer temperature and affect their lifetime [7], [8].

\section{Voltage unbalance issue}

The connection of PV to low voltage distribution network is made in single phase form. Naturally, there is voltage unbalance because most households use single-phase electrical appliances and the lines are not always transposed as they are in the case of transmission network. In practice, the load may be well distributed so that the powers circulating on different phases are the same and therefore the network is close to equilibrium. The injection of single-phase PV is a source of unbalance if it is not well distributed [9]. That is why DSO limit the minimal power of single-phase connected DG to quite lower values than three-phase connected ones. There is more than one definition of voltage unbalance [10]. We have used a Voltageunbalancefactor (2) that is derived from Fortescue transformation.

$$
\% \text { Voltageunbalance factor }(V U F)=\frac{V_{\text {neg }}}{V_{\text {pos }}} \times 100
$$

Where the negative sequence component $V_{n e g}$ and the positive sequence component $V_{\text {pos }}$ are given by (3) and (4):

$$
\begin{aligned}
& V_{n}=\frac{V_{a b}+a^{2} \cdot V_{b c}+a \cdot V_{c a}}{3} \\
& V_{p}=\frac{V_{a b}+a \cdot V_{b c}+a^{2} \cdot V_{c a}}{3}
\end{aligned}
$$

where $a=1 \angle 120^{\circ}$ and $a^{2}=1 \angle 240^{\circ}$ and $V_{i j}$ is the phase to phase voltage calculated in $p u$.

\section{Neutral current}

The considered LV distribution is a three-phase, four-wires system. The neutral current is a vector sum of the three line-toneutral current. In perfectly balanced power distribution, this current is nul. In low voltage distribution network supplying single-phase load, there is phase current unbalance and so a resulting neutral current [11].

In LV distribution system, neutral conductors are sized for a nominal current intensity. Injection of single-phase residential PV may increase the current phase unbalance and therefore the neutral current amplitude which can exceed a conductor limit [12].

\section{CHOSEN PLF METHOD}

The point estimate method allows to compute a moment of random variable that is the function of $m$ random variable inputs.

The power flow can be mathematically modeled as a set of non linear function $F_{i}$ that relates random parameters $p_{i}$ (bus power injection? line parameters...) and network electrical quantity $Z_{i}$ (bus voltage and angle, power losses in line, current...) (5).

$$
Z_{i}=F_{i}\left(p_{1}, p_{2}, p_{3}, \ldots, p_{m}\right)
$$

In our study case, we consider uncertainty on load consumption and PV generators output at a given moment.

We have used the point estimate method developed in [14][15]. It is based on the use of few statistical moments (mean, variance, skewness and kurtosis) of the random input parameters $p_{i}$ to compute the moments of the random variable $Z_{i}$. There are different types of point estimate method depending on the number of needed standard moments. For example, for the $2 m$ scheme, we just need mean, variance and skewness to perform it while the $3 m$ scheme requires mean, variance, skewness and kurtosis.

Each input random parameter $p_{l}$, with $l \in 1, \ldots, m$ is identified by its concentration which is a couple $p_{l, k}, w_{l, k}$, $k \in 1, \ldots, K$ which are respectively the location and the weight. $K$ is an integer number which depends on the chosen PEM type. At the first stage, we begin with $K=2$, which corresponds to the $2 m$ scheme. The location is given by (6) where $\mu_{p l}$ and $\sigma_{p l}$ are the mean and standard deviation of the input random variable. $\xi_{l, k}$ represents the standard location.

$$
p_{l, k}=\mu_{p l}+\xi_{l, k} \times \sigma_{p l}
$$


The standard location and the weight are obtained by solving the non linear system of equation [15] where $\lambda_{l, k}$ is the jth standard moment of the random variable $p_{l}$.

$$
\left\{\begin{array}{rlr}
\sum_{k=1}^{K} w_{l, k} & =1 \\
\sum_{k=1}^{K} w_{l, k}\left(\xi_{l, k}\right)^{j} & =\lambda_{l, k}
\end{array}\right.
$$

with $j=1,2, \ldots, 2 K-1$.

Equation (5) is computed $K$ times for each parameter $p_{l}$. For each evaluation, the parameter $p_{l}$ is set equal to its location $p_{l, k}$ and others parameters are represented by their mean $(\mu)$, see (8).

$$
Z(l, k)=F\left(\mu_{p 1}, \mu_{p 2}, \mu_{p 3}, \ldots, p_{l, k}, \ldots, \mu_{p m}\right)
$$

The deterministic load flow is computed using OpenDSS [20]. The calculation principle described in [16] is based on an iterative method which computes voltages from system admittance matrix and injection current of power converter elements (loads and generators).

By using the evaluated $Z(l, k)$ associated to the weighting factors $w_{l, k}$, the jth moment of the load flow output random variable $Z$ is estimated by (9)

$$
E[Z]^{j} \simeq \sum_{l=1}^{m} \sum_{k=1}^{K} w_{l, k}\left(Z(l, k)^{j}\right)
$$

\section{Simulation}

\section{A. Network Test}

We have used a ckt5 network test which is one of the benchmark test networks developed by EPRI [17]. These models are representative of actual small, medium and large circuit from various utilities. The network test cover a voltage range from $115 \mathrm{KV}$ to $208 \mathrm{~V}$ and serves 1379 household customers with a photo voltaic generation units on their roof. The one-line diagram of the test feeder is shown in (figure 1). The household position is set arbitrary through all the network.

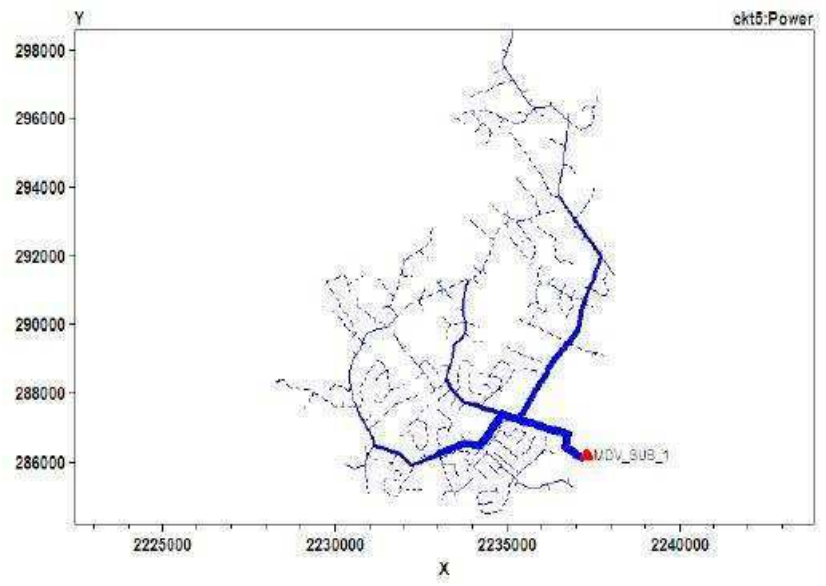

Fig. 1. One-line diagram of network test feeder

\section{B. Input data}

The input of our model, we need the probability density functions of considered input random parameters which are: the power delivered by PV and load consumption. From those pdf we determine statistical moments (mean, standard deviance, skewness) of each parameter.

1) Load model: We have considered 1379 single-phase loads. They are random parameters which are modeled by a probability density function of their active power. We suppose that the power factor is fixed and all the loads have the same pdf type.

The probability density functions of loads active power are build from house consumption data available in pecanstreet project [18]. The sets of data are power consumption measured during one year with one hour time step. So, for each hour, we have 365 measured values for active power. Data are classified by season and then fitted to a probability density function using a matlab pdf fitting function (figure 2). So each load's active power, at each hour is modeled by a generalized extreme value distribution. Mean value for all loads varies from $167 \mathrm{~W}$ to $5 k W$.

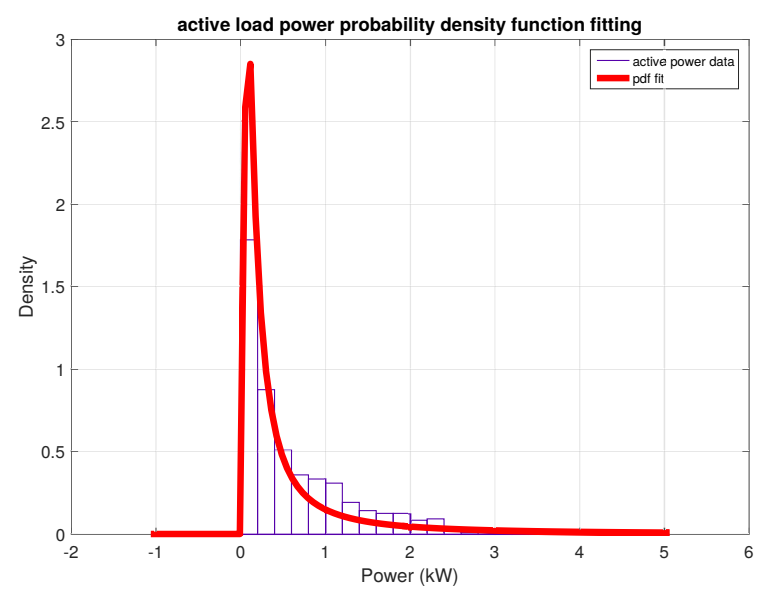

Fig. 2. Probability density function of active power of load 1 at $15 \mathrm{~h}$

2) PV production model: The electrical power from a photovoltaic generation unit at each time can be expressed by (10) where $I(t)$ is the clear sky irradiance $\left(W / m^{2}\right)$ at time $t$ depending on the inclination of the panel, $S$ the panel installed surface $\left(\mathrm{m}^{2}\right)$ and $\eta$ the panel efficiency. We model $1379 \mathrm{PV}$ units which are connected at the same nodes than the household load and randomly distributed through the grid. All PV panels are supposed to have the same efficiency $\eta$ which is set equal to $12 \%$. For each house, the installed panels surface is a uniformly pseudo random distributed integer varying from 5 to $10 \mathrm{~m}^{2}$.

$$
P_{p v}(t)=(1-c(t)) \times I(t) \times S \times \eta
$$

$c(t)$ is a random parameter which varies from 0 to 1 . It models a sky clear index, at each time $t$, which impact directly the photovoltaic production. The presence of clouds implies 
high value of $c$ (around 1) and so low PV production. In contrary, low value of $c$ (around 0) reflects a perfect clear sky and therefore a maximum PV production.

The random character of the PV production due to its irregularity is modeled here by the clear index $c(t)$. The PDF of this clear index is estimated from measured clear index data available in SCE project. The fitting process applied to those data lead to conclusion that beta distribution is the best suited as it shown in (figure 3).

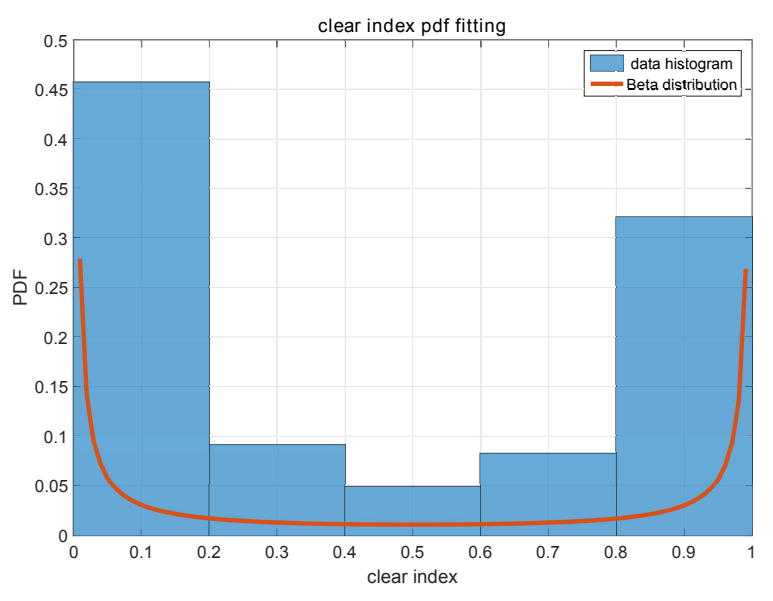

Fig. 3. Probability density function of sky clear index

\section{Results}

1) Average value: The prosed PEM method is applied to the network test using openDSS combined with Matlab. In PLF studies, results from Monte Carlo simulation are taken as reference to verify accuracy of others methods. That's why in this work, estimated voltages, currents and powers are compared to the estimations obtained from a Monte Carlo simulation with 10000 samples. High number of Monte Carlo sampling is chosen to guarantee a good accuracy of the estimated value. Those values are also compared to results from a deterministic load flow. The DLF is computed with hypothesis that active power loads consumption and PV generation are known perfectly and equal to the mean of random input parameters.

Figure 4, 5, 6 show estimated mean of nodes voltage, one phase conductor and neutral current through lines. For a good plot readability, we selected only few number of lines and nodes. In order to check accuracy of the PEM method, we define a relative error indice for each output estimated random value $Z$.

$$
\begin{aligned}
& E_{\mu}^{Z}=\left|\frac{\mu_{M C}^{Z}-\mu_{P E M}^{Z}}{\mu_{M C}^{Z}}\right| \\
& E_{\sigma}^{Z}=\left|\frac{\sigma_{M C}^{Z}-\sigma_{P E M}^{Z}}{\sigma_{M C}^{Z}}\right|
\end{aligned}
$$

$E_{\mu}^{Z}$ and $E_{\sigma}^{Z}$ represents respectively error in the mean and standard deviation of the output random variable $Z$. As explained before, $Z$ can be a node voltage, line current or neutral current

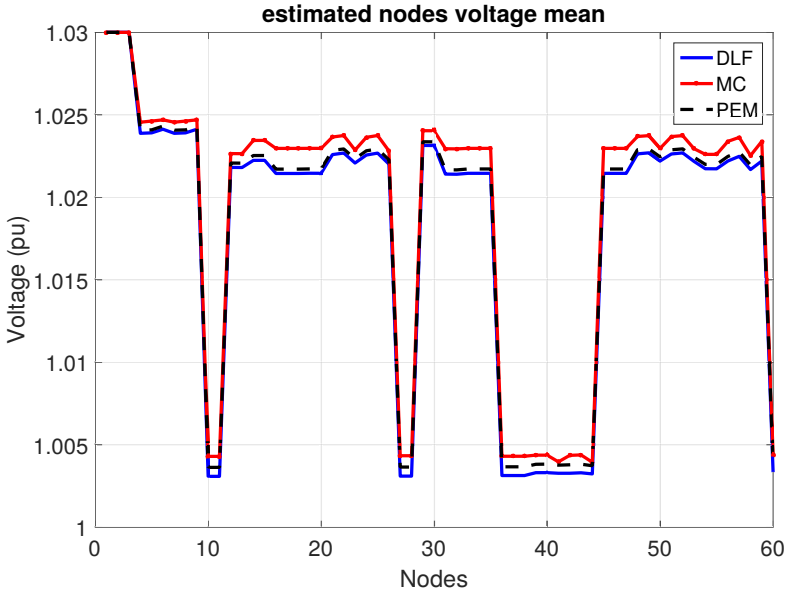

Fig. 4. Estimated mean of nodes voltage

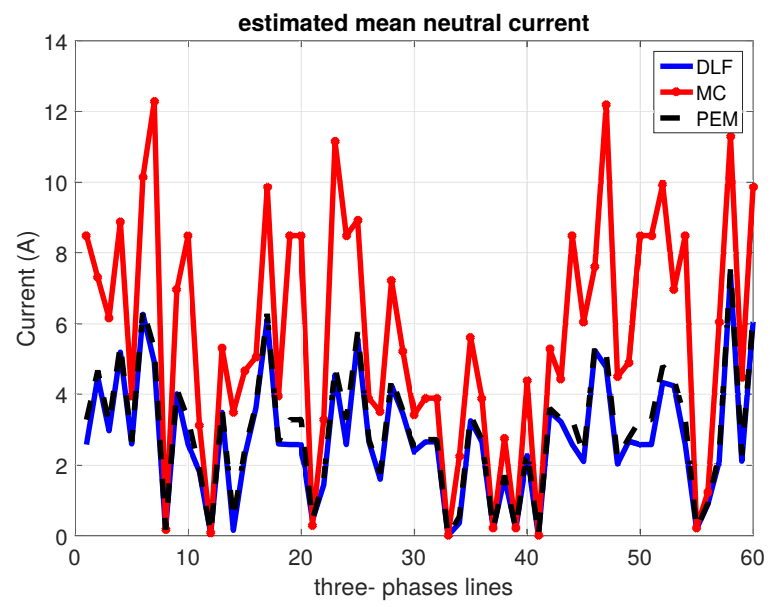

Fig. 5. Estimated mean of neutral lines current

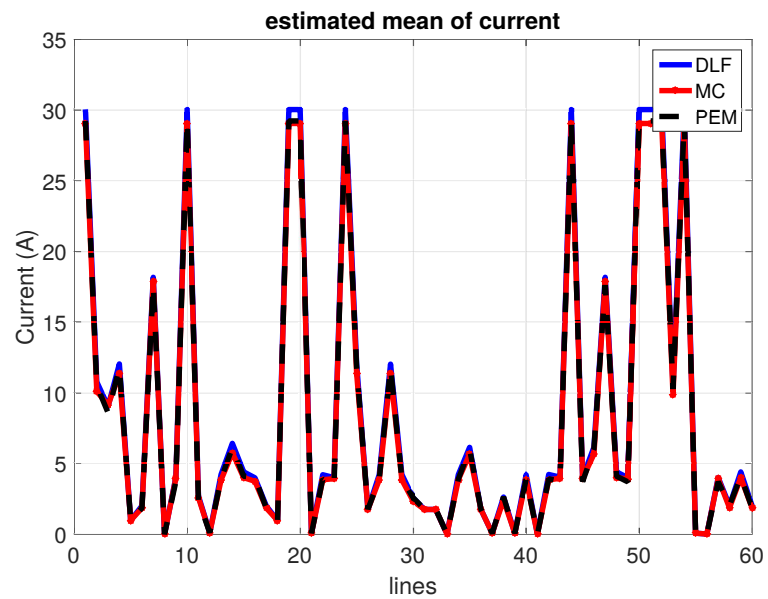

Fig. 6. Estimated mean of phases current

value. $\mu_{M C}^{Z}$ and $\sigma_{M C}^{Z}$ are mean and standard deviation values obtained from MCs, which is taken as reference. In the same 
way, $\mu_{P E M}^{Z}$ and $\sigma_{P E M}^{Z}$ are mean and standard deviation value estimated from the PEM method. Figure 7 shows error indice (in $p u$ ) on mean of estimated value for all nodes and lines. Figure 7 shows that the voltage mean estimation error for
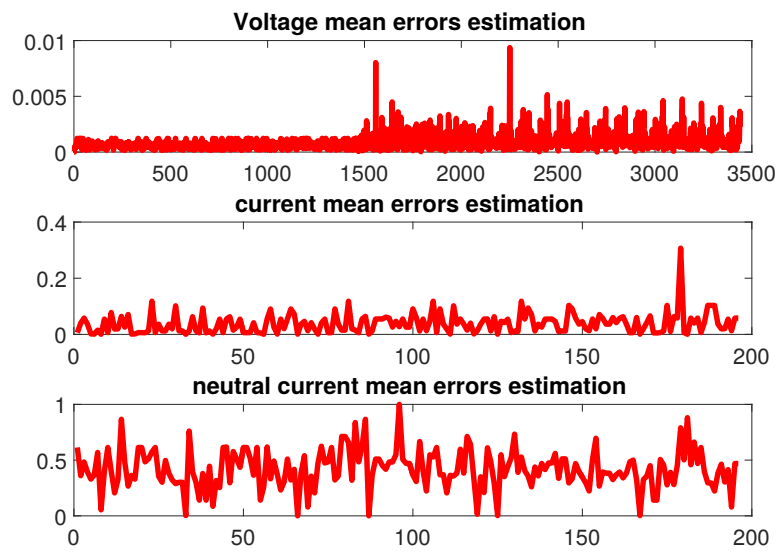

Fig. 7. Error indices between mean values estimated from PEM method and MC method

all electrical network nodes is less than $1 \%$. Average phase conductor current mean estimation is equal to $12 \%$ and it is noticed that neutral current one reaches $40 \%$. By integrating a Kurtosis coefficient in a PEM method, i.e choosing $(K=3)$, we notice a net decreasing of this average estimation error. Table I sums the averages estimation error between Monte Carlo method and PEM method with $K=2$ and $K=3$. This can be explained by the fact that the concentrations in PEM method with $K=2$ depends on the number of random parameters; that is not the case for $(K=3)$ PEM scheme. So the larger the size of the problem is, the less accurate the $(K=2)$ method is. Here we have 1380 random parameters. To my knowledge the largest number of parameters have seen in the literature corresponds to 170 parameters [15].

TABLE I

AVERAGE ESTIMATION ERRORS OF VOLTAGE, CURRENT AND NEUTRAL CURRENT MEAN VALUE

\begin{tabular}{|c|c|c|c|}
\hline PEME scheme & $V$ & $I$ & $I_{n}$ \\
\hline PEM $(K=2)$ & 0.0025 & 0.2862 & 0.3476 \\
\hline PEM $(K=3)$ & 0.0013 & 0.0837 & 0.1774 \\
\hline
\end{tabular}

2) Probability distribution: The mean and standard deviation presented above does not reflect variation of the estimated element and are insufficient to have any idea on the potential risk to have any voltage or current violation. To take into account the possible variation of estimated element, and therefore be able to quantify the risk to be out of the normal network operating limit, it is necessary to get the probability density function of this element. The displayed results are those from a bus which supply four houses connected to its phase C. We call it bus B.

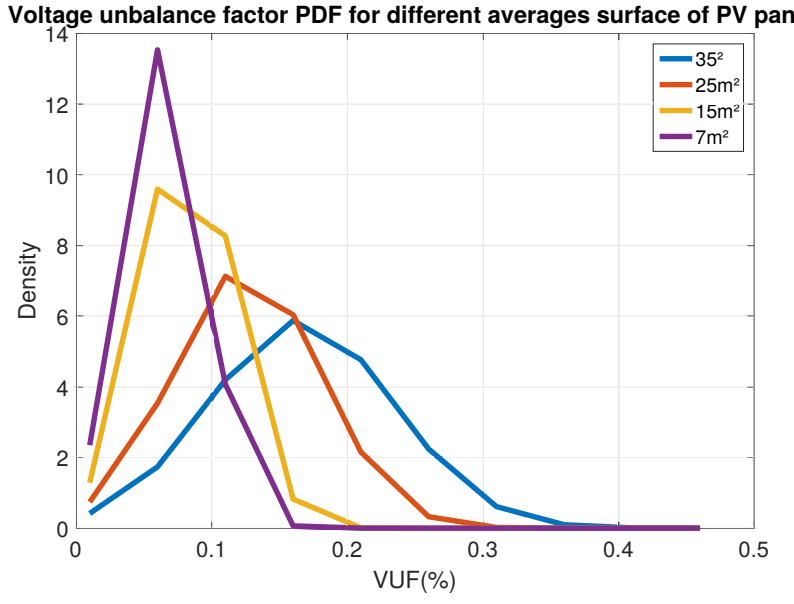

Fig. 8. Probability density function of voltage unbalance factory at a choosen bus $\mathrm{B}$

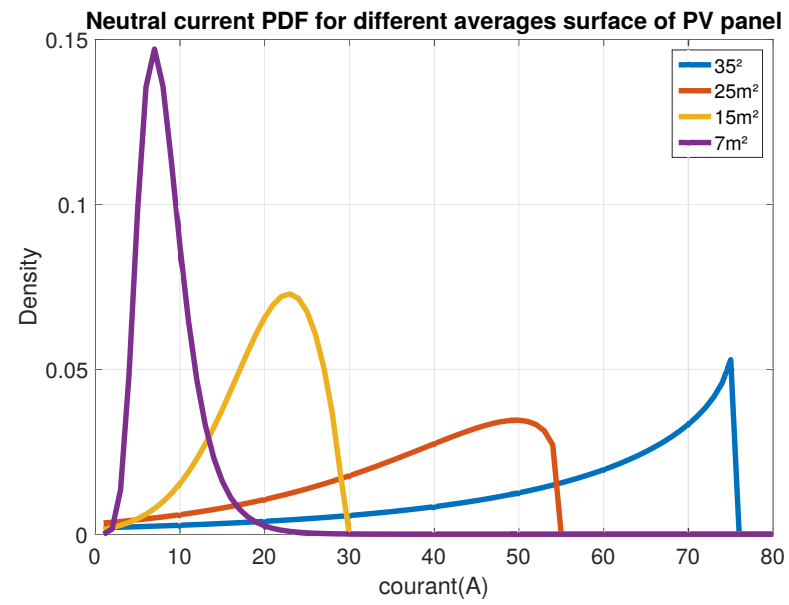

Fig. 9. Probability density function of neutral current in line connected to bus $\mathrm{B}$

\section{CONCLUSION}

This work aims to apply known PLF methods (Point estimate and Monte Carlo) on an unbalanced low voltage distribution network to estimate the impact of distributed generators on voltage, neutral current, phase current and voltage unbalance factor. A generalized extreme value and beta distribution have been used to model the random behavior of active power load consumption and photovoltaic power generation instead of normal distribution which is usualy used. Although results show an acceptable error between voltage mean estimated from PEM method and MC, a high error indice is noticed for the estimation of phase conductor and neutral current mean. Average errors for these two last elements are $40 \%$ for neutral current and $12 \%$ for phase conductor current. Those errors are decreased when using the $(2 m+1)$ PEM scheme (i.e $K=3$ ) which is more suitable for large size problem. The accuracy of the PEM method is really dependent on the size of the applied network, for this reason Monte Carlo 
simulation is more suitable in this case even if it requires 4 times simulation duration.

\section{ANNEXE}

Here follow, the used point estimate method flow chart as it's developed by the work in [15].

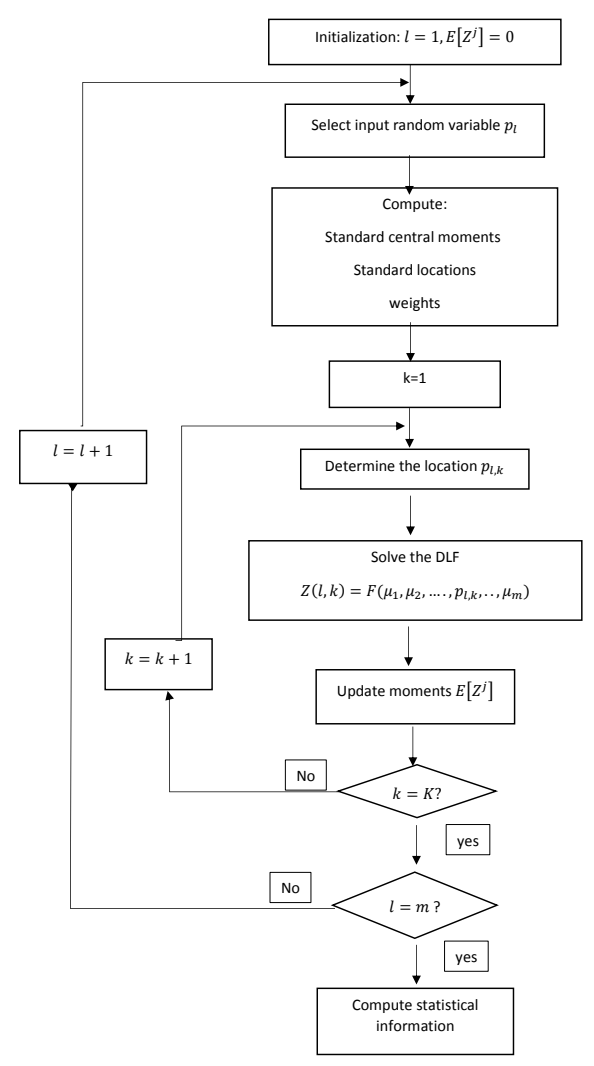

Fig. 10. Flow diagram of the algortihme.

\section{ACKNOWLEDGMENT}

This work is part of the project Smart City Energy analytics funded and managed by the IRTSystem $\mathrm{X}^{1}$.

\section{THE REFERENCES SECTION}

\section{REFERENCES}

[1] Conti, S., Raiti, S. (2007, May). Probabilistic load flow for distribution networks with photovoltaic generators part 1: Theoretical concepts and models. In 2007 International Conference on Clean Electrical Power (pp. 132-136). IEEE.

[2] Conti, S., Raiti, S., Di Gregorio, C. (2007, May). Probabilistic load flow for distribution networks with photovoltaic generators part 2: application to a case study. In 2007 International Conference on Clean Electrical Power (pp. 137-141). IEEE.

\footnotetext{
${ }^{1}$ http://www.irt-systemx.fr/project/sce/
}

[3] Dong, L., Lao, L., Yang, Y., Luo, J. (2011, March). Probabilistic load flow analysis considering power system random factors and their relevance. In Power and Energy Engineering Conference (APPEEC), 2011 Asia-Pacific (pp. 1-4). IEEE.

[4] Kaffashan, I., Amraee, T. (2015). Probabilistic undervoltage load shedding using point estimate method. IET Generation, Transmission Distribution, 9(15), 2234-2244.

[5] Begovic, M. M., Kim, I., Novosel, D., Aguero, J. R., Rohatgi, A. (2012, January). Integration of photovoltaic distributed generation in the power distribution grid. In System Science (HICSS), 2012 45th Hawaii International Conference on (pp. 1977-1986). IEEE.

[6] He, Y., Petit, M., Dessante, P. (2012, October). Optimization of the steady voltage profile in distribution systems by coordinating the controls of distributed generations. In 2012 3rd IEEE PES Innovative Smart Grid Technologies Europe (ISGT Europe) (pp. 1-7). IEEE.

[7] Mohammadi, P., Mehraeen, S. Challenges of PV Integration in LowVoltage Secondary Networks.

[8] Beaude, O., Lasaulce, S., Hennebel, M., Daafouz, J. (2015, July). Minimizing the impact of EV charging on the electricity distribution network. In Control Conference (ECC), 2015 European (pp. 648-653). IEEE.

[9] Chandra, S., Twomey, P., Randles, D. (2013). Impact of PV and load penetration on LV network voltages and unbalance and potential solutions. In Electricity Distribution (CIRED 2013), 22nd International Conference and Exhibition on (pp. 1-4). IET.

[10] Pillay, P., Manyage, M. (2001). Definitions of voltage unbalance. IEEE Power Engineering Review, 21(5), 50-51.

[11] Gruzs, T. M. (1990). A survey of neutral currents in three-phase computer power systems. IEEE Transactions on Industry Applications, 26(4), 719-725.

[12] Alam, M. J. E., Muttaqi, K. M., Sutanto, D. (2015). Alleviation of neutral-to-ground potential rise under unbalanced allocation of rooftop PV using distributed energy storage. IEEE Transactions on Sustainable Energy, 6(3), 889-898.

[13] Janecek, E., Georgiev, D. (2012). Probabilistic extension of the backward/forward load flow analysis method. IEEE Transactions on Power Systems, 27(2), 695-704.

[14] Su, C. L. (2005). Probabilistic load-flow computation using point estimate method. IEEE Transactions on Power Systems, 20(4), 18431851.

[15] Morales, J. M., Perez-Ruiz, J. (2007). Point estimate schemes to solve the probabilistic power flow. IEEE Transactions on Power Systems, 22(4), 1594-1601.

[16] Nie, S., Fu, X. P., Li, P., Gao, F., Ding, C. D., Yu, H., Wang, C. S. (2012, May). Analysis of the impact of DG on distribution network reconfiguration using OpenDSS. In IEEE PES Innovative Smart Grid Technologies (pp. 1-5). IEEE.

[17] IEEE Distribution Test feeder http://ewh.ieee.org/soc/pes/dsacom/ testfeeders/, 12/12/2016

[18] Pecanstreet project http://www.pecanstreet.org/, 12/12/2016

[19] Chassin, D. P., Schneider, K., Gerkensmeyer, C. (2008, April). GridLAB-D: An open-source power systems modeling and simulation environment. In 2008 IEEE/PES Transmission and Distribution Conference and Exposition.

[20] Dugan, R. C. (2012). Reference guide: The open distribution system simulator (opendss). Electric Power Research Institute, Inc. 